Original paper

\title{
Relation between levels of toll-like receptors 3 and 7 and clinical profile of Child-Pugh B cirrhotic patients
}

\author{
Ahmed Kamal', Ahmed Ramadan², Dalia Sherief ${ }^{3}$, Asmaa Hassan ${ }^{3}$, Nahla Nosair ${ }^{3}$, Maaly Mabrouk4 ${ }^{4}$ Eman Habeeb3 \\ Tamer Haydra ${ }^{5}$ \\ 'Internal Medicine Department, Hepatology Unit, Faculty of Medicine, Alexandria University, Egypt \\ 2Department of Demography And Biostatistics, Faculty of Graduate Studies for Statistical Research, Cairo University, Egypt \\ ${ }^{3}$ Clinical Pathology Department, Faculty of Medicine, Kafr El-Sheikh University, Egypt \\ ${ }^{4}$ Clinical Pathology Department, Faculty of Medicine, Tanta University, Egypt \\ ${ }^{5}$ Internal Medicine Department, Faculty of Medicine, Kafr El-Sheikh University, Egypt
}

\begin{abstract}
Aim of the study: Growing data show that toll-like receptors (TLRs) have considerable roles in the pathogenesis of many liver diseases. We aimed to study the relation between TLR3 and TLR7 levels and clinical manifestations of liver decompensation among hepatitis C virus (HCV)-infected Child-Pugh B patients.

Material and methods: This study included 60 adult patients with Child-Pugh B liver cirrhosis on top of untreated HCV infection. We performed a two-step clustering algorithm depending on TLR-3 gene expression, TLR-7 gene expression, and other influential patients' characteristics.

Results: Patients were optimally divided into 2 clusters, each cluster containing 30 patients. The average silhouette score of the clustering algorithm was 0.52 , indicating a good clustering power of the model. Patients in cluster 1 showed lower relative expression of TLR3 (0.188 vs. 0.29). The same was true of TLR7 (0.20 vs. 0.31$)$. All patients within cluster 1 had lower limb edema and $93 \%$ of them had ascites. On the other hand, no one within cluster 2 had ascites or lower limb edema. The mean platelet count was lower in patients within cluster 1 (74,000 vs. 100,000 cell/ $/ \mathrm{mm}^{3}$ ). The mean international normalized ratio (INR) level was higher in cluster 1 (1.61 vs. 1.3). The mean Model for End-Stage Liver Disease (MELD) score was higher in cluster 1 (15 vs. 10).

Conclusions: From these results, we can suggest that lower TLR3 and TLR7 can lead to worse clinical manifestations among patients with HCV-related liver cirrhosis. A deeper exploration of this point can open the door for new approaches for managing decompensated cirrhosis.
\end{abstract}

Key words: chronic hepatitis C, toll-like receptors, decompensated cirrhosis.

Address for correspondence:

Dr. Ahmed Kamal, Internal Medicine Department, Hepatology Unit, Faculty of Medicine, Alexandria University, Egypt, e-mail: ahmed.kamal@alexmed.edu.eg

\section{Introduction}

Hepatitis $\mathrm{C}$ virus (HCV) is one of the most prevalent causes of chronic liver diseases, which results in inflammatory and wound-healing responses leading to liver fibrogenesis and hepatocellular carcinoma (HCC) development $[1,2]$.

Toll-like receptors (TLRs) have a major role in body immunity through identifying pathogen-associated molecular patterns (PAMPs) [3]. Many studies have shown that TLRs have an important role in the pathogenesis of many liver diseases including viral hepatitis, non-alcoholic steatohepatitis (NASH), alcoholic hepatitis, autoimmune hepatitis, hepatic fibrogenesis, and liver carcinogenesis [4-8].

Decreased expression of TLR3 and TLR7 in cells infected with HCV has been shown [9-11]. Treatment with interferon $\alpha(\mathrm{IFN}-\alpha)$ was used to counteract this effect. 
In a study performed by Horsmans et al., treatment with intravenous isatoribine given as an agonist for TLR7 caused a significant decrease in HCV viral load [12]. TLR7 agonist was shown to decrease HCV viral load in another study [13].

TLR3 deficiency is also linked to the progression of liver fibrosis. Treatment of mice with the TLR3 ligand poly I:C was shown to inhibit liver fibrogenesis [14-16]. Also, TLR7 down-regulation was suggested to contribute to liver fibrosis progression [17].

We aimed to study the relation between TLR3 and TLR7 levels and clinical manifestations of liver decompensation among $\mathrm{HCV}$-infected Child-Pugh B patients.

\section{Material and methods}

\section{Materials}

This study included 60 adult patients with ChildPugh B liver cirrhosis on top of untreated HCV infection who were referred to the Internal Medicine Department, Kafr Elsheikh University Hospital. Patients with HBV or HIV co-infection and patients with hepatocellular carcinoma were excluded. Informed consent was obtained from all participants. Performance of the study was approved by Kafr Elsheikh Faculty of Medicine Ethical Committee.

\section{Methods}

Blood samples were collected from the included patients for evaluation of complete blood picture $(\mathrm{CBC})$, prothrombin time $(\mathrm{PT})$, international nor-

Table 1. Characteristics of the 2 clusters

\begin{tabular}{lcc}
\hline Item & Cluster 1 & Cluster 2 \\
\hline Mean age (years) & 46.67 & 52.07 \\
\hline Males (\%) & 53.3 & 53.3 \\
\hline Lower limb edema (\%) & 100 & 0 \\
\hline Ascites (\%) & 93.3 & 0 \\
\hline Mean relative levels of TLR-3 gene expression & 0.19 & 0.29 \\
\hline Mean relative levels of TLR-7 gene expression & 0.21 & 0.31 \\
\hline Mean INR level & 1.61 & 1.30 \\
\hline Mean platelet count (cell/mm ${ }^{3}$ ) & 74.27 & 100.73 \\
\hline Mean total leucocyte count (cell/mm $\left.{ }^{3}\right)$ & 2.36 & 3.63 \\
\hline Mean total bilirubin level (mg/dl) & 1.17 & 1.06 \\
\hline Mean hemoglobin level (g/dl) & 8.47 & 9.53 \\
\hline Mean creatinine level (mg/dl) & 1.66 & 0.97 \\
\hline Mean FIB-4 score & 5.17 & 4.27 \\
\hline Mean MELD score & 15.2 & 10.79 \\
\hline
\end{tabular}

malized ratio (INR), liver and kidney function tests, HBs antigen, anti-HCV antibody by enzyme-linked immunosorbent assay (ELISA), and serum $\alpha$-fetoprotein (AFP) levels. HCV RNA was assayed by reverse transcription-polymerase chain reaction (RT-PCR) to confirm ongoing viremia.

Determination of relative levels of TLR3 and TLR7 expression [18]: Relative levels of TLR3 and TLR7 gene expression were determined by Rotor-Gene Q RealTime PCR instruments from QIAGEN USA. RNA was extracted by a QIAamp RNA Blood Mini Kit (Qiagen, Germany) on a QIAcube (Germany). After this, mRNA was transcribed into cDNA using the Omniscript reverse transcription kit (Qiagen, Dusseldorf, Germany). To quantify the relative expression of each gene, the comparative $\Delta \Delta \mathrm{Ct}$ method was used.

Clustering algorithm [19]: A two-step clustering algorithm was performed depending on: age, gender, presence of lower limb edema, presence of ascites, INR, platelets, total leucocyte count, hemoglobin, total bilirubin, creatinine levels, FIB-4 score [20], Model for End-Stage Liver Disease (MELD) score [21], TLR-3 gene expression and TLR-7 gene expression.

\section{Results}

Patients were optimally divided into 2 clusters, each cluster containing 30 patients. The average silhouette score of the clustering algorithm was 0.52 , indicating a good clustering power of the model. Patients in cluster 1 showed lower relative expression of TLR3 (0.188 vs. 0.29$)$. The same was true of TLR7 ( 0.20 vs. 0.31 ). All patients within cluster 1 had lower limb edema and $93 \%$ of them had ascites. On the other hand, no one within cluster 2 had ascites or lower limb edema. The mean platelet count was lower in patients within cluster $1\left(74,000\right.$ vs. 100,000 cell $\left./ \mathrm{mm}^{3}\right)$; the same was true of mean hemoglobin levels ( $8.4 \mathrm{vs} .9 .5 \mathrm{~g} / \mathrm{dl})$ and total leucocyte count $\left(2.36\right.$ vs. $3.6 \times 10^{3}$ cell $\left./ \mathrm{mm}^{3}\right)$. Mean INR level was higher in cluster 1 (1.61 vs. 1.3$)$ and the same was true of creatinine $(1.66 \mathrm{vs} .0 .97 \mathrm{mg} / \mathrm{dl})$. The mean MELD score was higher in cluster 1 ( 15 vs. 10$)$. Interestingly, the mean FIB-4 score was higher in cluster 1 (5.17 vs. 4.27 ) (Table 1 ).

\section{Discussion}

Toll-like receptors activate the innate immune system through the identification of PAMPs [3]. TLRs also play roles in activation of the adaptive immune system, regulation of inflammatory responses, cellular regeneration, and carcinogenesis $[2,4,9,14]$. 
The ability of HCV to inhibit the immune response aiming to maintain its persistence has been revealed [13]. Decreased expression of TLR3 and TLR7 in cells infected with HCV via inhibitory effects of $\mathrm{HCV}$ proteins aiming to prevent the immune system from clearing the virus has been shown $[17,18]$. Treatment with IFN- $\alpha$ was used to counteract this effect.

Moreover, deficiency of TLR3-mediated natural killer (NK) cell-dependent apoptosis of hepatic stellate cells (HSCs) has been associated with the progression of liver fibrogenesis in patients with alcoholic hepatitis [23]. It was suggested that treatment with the TLR3 ligand poly I:C induces NK cells which promote apoptosis of HSCs [24-26].

Lower expression of TLR7 was found in malignant hepatocytes compared with non-malignant hepatocytes among patients with HCC [27]. Also, phosphorothioate-modified TLR9 agonist was suggested to have anti-HCC activity [28]. Strangely, another trial reported different results. This trial showed that TLR7 and TLR9 were overexpressed in the HCC biopsies compared with biopsies from patients with liver cirrhosis but without HCC. The authors suggested that the inhibition of TLR7 and TLR9 with IRS-954 or chloroquine might be used for HCC prevention in susceptible patients [29].

We aimed to study the levels of TLR3 and TLR7 in patients with HCV-related decompensated cirrhosis lying within Child-Pugh class B and to determine if there is a relation between their levels and clinical manifestations of patients.

In our clusters, $100 \%$ of patients among the cluster with lower TLR3 and TLR7 had lower limb edema and 93\% had ascites, while no patient within the other cluster had lower limb edema or ascites. Also, lower TLR3 and TLR7 levels were associated with lower albumin, hemoglobin, platelets, and total leucocyte count. Also, lower TLR3 and TLR7 levels were associated with higher INR, total bilirubin, FIB-4, and MELD scores.

From these results, we can suggest that lower TLR3 and TLR7 can lead to worse clinical manifestations among patients with HCV-related liver cirrhosis. These findings need to be validated on a larger population and to be explored among patients with treated HCV. Also, the pathogenesis of these findings needs to be investigated. This can open the door for new approaches for managing decompensated cirrhosis through studying the effect of TLR3 and TLR7 agonists on the clinical outcomes of these patients.

\section{Disclosure}

The authors declare no conflict of interest.

\section{References}

1. Gomaa A, Allam N, Elsharkawy A, et al. Hepatitis C infection in Egypt: prevalence, impact and management strategies. Hepat Med 2017; 9: 17-25.

2. Cordero-Espinoza L, Huch M. The balancing act of the liver: tissue regeneration versus fibrosis. J Clin Investig 2018; 128: 8596.

3. El-Zayat SR, Sibaii H, Mannaa FA. Toll-like receptors activation, signaling, and targeting: an overview. Bull Natl Res Cent 2019; 43: 187.

4. Kesar V, Odin JA. Toll-like receptors and liver disease. Liver Int 2014; 34: 184-196.

5. Neamatallah M, El-Bendary M, Elalfy H, et al. Impact of tolllike receptors 2 (TLR2) and TLR4 gene variations on HCV susceptibility, response to treatment and development of hepatocellular carcinoma in cirrhotic HCV patients. Immunol Invest 2020; 49: 462-476.

6. Khambu B, Yan S, Huda N, Yin XM. Role of high-mobility group box-1 in liver pathogenesis. Int J Mol Sci 2019; 20: 5314.

7. Nasiri M, Karimi MH, Azarpira N, et al. Gene expression profile of toll-like receptor/adaptor/interferon regulatory factor/cytokine axis during liver regeneration after partial ischemia-reperfusion injury. Exp Clin Transplant 2020; 18: 215-223.

8. Tong J, Guo JJ. Key molecular pathways in the progression of non-alcoholic steatohepatitis. Eur Rev Med Pharmacol Sci 2019; 23: 8515-8522.

9. Moradi M, Tabibzadeh A, Javanmard D, et al. Assessment of key elements in the innate immunity system among patients with HIV, HCV, and coinfections of HIV/HCV. Curr HIV Res 2020; 18: 194-200.

10. Kar P, Kumar D, Gumma PK, et al. Down regulation of TRIF, TLR3, and MAVS in HCV infected liver correlates with the outcome of infection. J Med Virol 2017; 89: 2165-2172.

11. Motavaf M, Noorbakhsh F, Alavian SM, et al. Distinct toll-like receptor 3 and 7 expression in peripheral blood mononuclear cells from patients with chronic hepatitis $\mathrm{C}$ infection. Hepat Mon 2014; 14: el6421.

12. Horsmans Y, Berg T, Desager JP, et al. Isatoribine, an agonist of TLR7, reduces plasma virus concentration in chronic hepatitis C infection. Hepatology 2005; 42: 724-731.

13. Funk E, Kottilil S, Gilliam B, et al. Tickling the TLR7 to cure viral hepatitis. J Transl Med 2014; 12: 129.

14. Jeong WI, Park O, Radaeva S, et al. STAT1 inhibits liver fibrosis in mice by inhibiting stellate cell proliferation and stimulating NK cell cytotoxicity. Hepatology 2006; 44: 1441-1451.

15. Radaeva S, Sun R, Jaruga B, et al. Natural killer cells ameliorate liver fibrosis by killing activated stellate cells in NKG2Ddependent and tumor necrosis factor-related apoptosis-inducing ligand-dependent manners. Gastroenterology 2006; 130: 435-452.

16. Krizhanovsky V, Yon M, Dickins RA, et al. Senescence of activated stellate cells limits liver fibrosis. Cell 2008; 134: 657-667.

17. El Aggan H, Farahat N, El Deeb N, et al. Peripheral blood and hepatic Toll-like receptor 7 expression and interferon lambda 1 levels in chronic hepatitis C: Relation to virus replication and liver injury. Microb Pathog 2019; 131: 65-74.

18. Sanders R, Mason DJ, Foy CA, et al. Considerations for accurate gene expression measurement by reverse transcription quantitative PCR when analysing clinical samples. Anal Bioanal Chem 2014; 406: 6471-6483. 
19. Garcia D, MacDonald S, Archer T. Two different approaches to the affective profiles model: median splits (variable-oriented) and cluster analysis (person-oriented). PeerJ. 2015; 3: e1380.

20. Vallet-Pichard A, Mallet V, Nalpas B, et al. FIB-4: an inexpensive and accurate marker of fibrosis in HCV infection. comparison with liver biopsy and fibrotest. Hepatology 2007; 46: 32-36.

21. Peng Y, Qi X, Guo X. Child-Pugh versus MELD score for the assessment of prognosis in liver cirrhosis: a systematic review and meta-analysis of observational studies. Medicine (Baltimore) 2016; 95: e2877.

22. Kawai T, Sato S, Ishii KJ, et al. Interferon-alpha induction through Toll-like receptors involves a direct interaction of IRF7 with MyD88 and TRAF6. Nat Immunol 2004; 5: 1061-1068.

23. Weiskirchen R, Tacke F. Cellular and molecular functions of hepatic stellate cells in inflammatory responses and liver immunology. Hepatobiliary Surg Nutr 2014; 3: 344-363.

24. Scott MJ, Billiar TR. Beta2-integrin-induced p38 MAPK activation is a key mediator in the CD14/TLR4/MD2-dependent uptake of lipopolysaccharide by hepatocytes. J Biol Chem 2008; 283: 29433-29446.

25. Liu S, Gallo DJ, Green AM, et al. Role of toll-like receptors in changes in gene expression and NF-kappa B activation in mouse hepatocytes stimulated with lipopolysaccharide. Infect Immun 2002; 70: 3433-3442.

26. Thobe BM, Frink M, Hildebrand F, et al. The role of MAPK in Kupffer cell toll-like receptor (TLR)2-, TLR4-, and TLR9-mediated signaling following trauma-hemorrhage. J Cell Physiol 2007; 210: 667-675.

27. Abdel-Raouf TA, Ahmed A, Zaki WK, et al. Study of toll-like receptor 7 expression and interferon $\alpha$ in Egyptian patients with chronic hepatitis $\mathrm{C}$ infection and hepatocellular carcinoma. Egypt J Med Hum Genet 2014; 15: 387-392.

28. Zhang Y, Lin A, Zhang C, et al. Phosphorothioate-modified $\mathrm{CpG}$ oligodeoxynucleotide (CpG ODN) induces apoptosis of human hepatocellular carcinoma cells independent of TLR9. Cancer Immunol Immunother 2014; 63: 357-367.

29. Mohamed FE, Al-Jehani RM, Minogue SS, et al. Effect of tolllike receptor 7 and 9 targeted therapy to prevent the development of hepatocellular carcinoma. Liver Int 2015; 35: 1063 1076. 